\title{
Narrow escape for a stochastically gated Brownian ligand
}

\author{
Jürgen Reingruber and David Holcman \\ Department of Computational Biology, Ecole Normale Supérieure, 46 rue d'Ulm, \\ 75005 Paris, France \\ E-mail: david.holcman@biologie.ens.fr
}

Received 8 September 2009, in final form 5 January 2010

Published 22 January 2010

Online at stacks.iop.org/JPhysCM/22/065103

\begin{abstract}
Molecular activation in cellular microdomains is usually characterized by a forward binding rate, which is the reciprocal of the arrival time of a ligand to a key target. Upon chemical interactions or conformational changes, a Brownian ligand may randomly switch between different states, and when target activation is possible in a specific state only, switching can significantly alter the activation process. The main goal of this paper is to study the mean time for a switching ligand to activate a small substrate, modelled as the time to exit a microdomain through a small absorbing window on the surface. We present the equations for the mean sojourn times the ligand spends in each state, and study the escape process with switching between two states in dimension one and three. When the ligand can exit in only one of the two states, we find that switching always decreases its sojourn time in the state where it can exit. Moreover, the fastest exit is obtained when the ligand diffuses most of the time in the state with the maximal diffusion coefficient, although this may imply that it spends most of the time 'hidden' in the state where it cannot exit. We discuss the physical mechanisms responsible for this apparent paradox. In dimension three we confirm our results with Brownian simulations. Finally, we suggest possible applications in cellular biology.
\end{abstract}

\section{Introduction}

The physiological response of cells to internal or external stimuli is often initiated by the binding of diffusing ligands (molecules, proteins, ions) to specific target activator proteins [1-6]. The time response depends on the mean time for the diffusing ligand to find these activators in the complex cellular environment [6-12]. The mean time for a diffusing ligand to find a small target molecule in a confined environment, known as the narrow escape time (NET), depends on the structure and geometry of the microdomain, and on the shape of the target. Explicit expressions for the NET in various situations are given in [13-21]. The NET has been used to derive statistical properties of chemical reactions with a few diffusing molecules [12], to obtain estimates for the probability and the arrival time of viral particles to nuclear pores [22, 23], to study receptor trafficking at synaptic membranes [24, 25], or to estimate the rate of cyclic GMP hydrolysis in rod photoreceptors [26].

However, when diffusing ligands switch between different states due to conformational changes or reversible chemical interactions with partners, in which case the switching rate depends on the concentration of these molecules, it is necessary to incorporate such situations into computations of the NET, especially when target activation is possible only in a specific state. For example, diffusing enzymes in the cytoplasm switch between an inactive and active state; in the nucleus, activation of a small DNA-site by a diffusing transcription factor (TF) is highly controlled by the interactions with various regulatory proteins changing the affinity of the TF to its promoter site [27-31].

Stochastic modellings of TF capture some of the complexity of the bio-molecular system. Usually the TF motion is approximated by the one of the centre of mass, but its segment motion and interactions with a target via appropriate residues is still not at hand. In the past, diffusion-limited chemical reactions have been studied when either the ligands or the target stochastically switch between different conformations [7, 32-36], and interestingly, it was found that the dynamics of the reactions differ between both scenarios. Gated ligands can be effectively modelled by a reduction in the reactivity, while for gated target all 


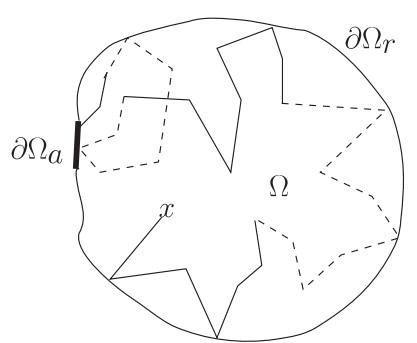

Figure 1. Schematic representation of a diffusing Brownian ligand that randomly switches between two states 1 (continuous line) and 2 (dashed line). In state 2 , the ligand is reflected all over the boundary of the microdomain $\Omega$, while in state 1 it is absorbed at $\partial \Omega_{\mathrm{a}}$.

ligands become correlated, and the survival probability of the target can change from exponential to non-exponential behaviour [33, 34, 37]. In a scenario where the target is surrounded by a fluctuating potential barrier, a resonant-like phenomena was found where the mean activation time has a minimum at a specific fluctuation rate $[38,39]$. Recently, certain classes of intermittent search processes were introduced and analysed [40-44], in which a ligand searches for a hidden target by stochastically switching between a fast ballistic and a slow diffusive phase, while the target can only be found in the diffusing phase. Interestingly, it was shown that switching decreases the search time, and, similar to a target activation over a fluctuating barrier, there are optimal switching rates that minimize the search time.

Here we study the gated narrow escape time (GNET), which is the time for a diffusing ligand that stochastically switches between different states with different diffusion coefficients to exit a microdomain $\Omega$ through a small window. We focus on a switching process that occurs between two states $(1,2)$ with diffusion coefficients $\left(D_{1}, D_{2}\right)$ (see figure 1$)$, where switching is described by

$$
1 \underset{k_{21}}{\stackrel{k_{12}}{\rightleftharpoons}} 2
$$

with Poissonian rates $\left(k_{12}, k_{21}\right)$. We investigate the cases when the ligand can exit only in state 1 , and in both states. To estimate the GNET, we do not use the equations for the mean first passage time, but we start from the equations for the sojourn times the ligand spends in each state before exiting, which provides a more detailed description of the switching process. The GNET is also related to intermittent search processes, where some switching strategies between different states lead to some minimal search time to find a target [40-43].

When the ligand can exit only in state 1 , all the mean exit and sojourn times can be expressed as a function of the sojourn time $u_{1}(1)$ the ligand spends in state 1 conditioned on starting in state 1 . If the domain $\Omega$ is the one-dimensional interval $(0, L)$, we find here that the exact solution $u(1)$ for the exit time starting initially in state 1 is (see equation (17))

$$
\begin{aligned}
u(1) & =\left(1+\frac{k_{12}}{k_{21}}\right) u_{1}(1)=\left(1+\frac{k_{12}}{k_{21}}\right) \frac{L^{2}}{3 D_{1}}\left(\frac{k_{21} D_{1}}{k_{12} D_{2}+k_{21} D_{1}}\right. \\
& \left.+\frac{3 k_{12} D_{2}}{k_{12} D_{2}+k_{21} D_{1}} f\left(\frac{k_{12} L^{2}}{D_{1}}+\frac{k_{21} L^{2}}{D_{2}}\right)\right)
\end{aligned}
$$

where $f(x)=\frac{\operatorname{coth} \sqrt{x}}{\sqrt{x}}-\frac{1}{x}$. In dimension three, we obtain the new asymptotic formula for the GNET $u(1)$ is (see equation (46))

$$
\begin{aligned}
u(1) & =\left(1+\frac{k_{12}}{k_{21}}\right) u_{1}(1)=\left(1+\frac{k_{12}}{k_{21}}\right) \\
& \times \begin{cases}\tau_{1}, & l_{1} \ll 1 \\
\frac{|\Omega|}{\left|\partial \Omega_{\mathrm{a}}\right| \sqrt{D_{1} k_{12}}}, & l_{1} \gg 1 \quad \text { or } \quad l_{2} \gg l_{1}\end{cases}
\end{aligned}
$$

where $\tau_{1}$ is the usual NET of a ligand that diffuses in state 1 without switching, $\left|\partial \Omega_{\mathrm{a}}\right|$ is the surface of the absorbing window, which is of the order $a^{2},|\Omega|$ is the volume of the domain, and $l_{1}=\frac{k_{12} a^{2}}{D_{1}}$ and $l_{2}=\frac{k_{21} a^{2}}{D_{2}}$ are the effective parameters that control the behaviour of $u(1)$. As we will see, our analysis reveals that switching not only drastically affects the exit time, but also, only for $D_{2}>D_{1}$, the GNET $u$ (1) has a minimum as a function of the switching rates. Interestingly, if switching occurs very fast, we find that a ligand can exit almost as fast as possible, although it spends most of the time in state 2 where exit is not possible, a phenomena that is related to the behaviour of the ligand in the boundary layer of the small absorbing site. When the ligand can exit in both states, our result for the exit time $u(1)$ in dimension three is (see equation (57))

$$
u(1)=\tau_{1} \frac{1+\left(k_{12}+k_{21}\right) \tau_{2}}{1+k_{12} \tau_{1}+k_{21} \tau_{2}},
$$

where $\tau_{2}=D_{1} / D_{2} \tau_{1}$ is the usual NET for a ligand that diffuses in state 2 without switching. Finally, we will suggest possible applications of our results in cellular biology.

\section{The equations for the sojourn times}

The dynamics of a Brownian particle diffusing in a bounded domain $\Omega$ and switching between $N$ independent states $n=$ $1, \ldots, N$ can be approximated by discrete stochastic dynamics

$$
\begin{aligned}
& X_{n}(t+\Delta t) \\
& \quad= \begin{cases}X_{m}(t) & \text { w.p. } k_{m n} \Delta t, \quad n \neq m \\
X_{m}(t)+\sqrt{2 D_{m} \Delta t} \eta & \text { w.p. } 1-k_{m}^{+} \Delta t, \quad n=m\end{cases}
\end{aligned}
$$

where $X_{n}(t)$ is the position of the particle in state $n, \eta$ a Gaussian variable, $D_{n}$ the diffusion coefficient in state $n, k_{n m}$ the transition rate from state $n$ to $m\left(k_{n n}=0\right)$, and $k_{n+}=$ $\sum_{i=1}^{N} k_{n i}$ is the cumulative rate to switch from state $n$ to any other state.

To further characterize the dynamics, we consider the transition probability density function $p\left(\boldsymbol{x}, n, t \mid \boldsymbol{y}, m, t^{\prime}\right)$ to find a particle that is initially at time $t^{\prime}$ in state $m$ at position $\boldsymbol{y}$, and at a later time $t$ in state $n$ at position $\boldsymbol{x}$. During the infinitesimal time interval $\tau=t-t^{\prime}$ and to first order in $\tau$, the dynamics can be decomposed into two distinct events (see equation (1)): first, switching from a state $n$ to a different state $m$ without diffusing, and second, diffusion from position 
$\boldsymbol{x}$ to position $\boldsymbol{y}$ without switching. From this we obtain for the infinitesimal transition probability density function

$$
\begin{aligned}
p(\boldsymbol{x}, & \left.n, t^{\prime}+\tau \mid \boldsymbol{y}, m, t^{\prime}\right)=k_{m n} \tau \delta(\boldsymbol{x}-\boldsymbol{y}) \\
& +\delta_{n m}\left(1-\tau k_{m+}\right)\left(1+\tau D_{m} \Delta_{x}\right) \delta(\boldsymbol{x}-\boldsymbol{y}) \\
= & \left(1-\tau k_{m+}\right) \delta_{n m} \delta(\boldsymbol{x}-\boldsymbol{y})+\tau\left(k_{m n}+\delta_{n m} D_{m} \Delta_{x}\right) \\
& \times \delta(\boldsymbol{x}-\boldsymbol{y})+\mathrm{O}\left(\tau^{2}\right) .
\end{aligned}
$$

To derive the forward Fokker-Planck equation we use equation (2) together with the Chapman-Kolmogorov equation $[45,46]$ and obtain

$$
\begin{aligned}
p(\boldsymbol{x}, & \left.n, t+\tau \mid \boldsymbol{y}, m, t^{\prime}\right)=\int_{\Omega} \mathrm{d} \boldsymbol{z} \sum_{i=1}^{N} p(\boldsymbol{x}, n, t+\tau \mid \boldsymbol{z}, i, t) \\
& \times p\left(\boldsymbol{z}, i, t \mid \boldsymbol{y}, m, t^{\prime}\right)=p\left(\boldsymbol{x}, n, t \mid \boldsymbol{y}, m, t^{\prime}\right)\left(1-\tau k_{n+}\right) \\
& +\tau \sum_{i=1}^{N} k_{i n} p\left(\boldsymbol{x}, i, t \mid \boldsymbol{y}, m, t^{\prime}\right) \\
& +\tau D_{n} \Delta_{x} p\left(\boldsymbol{x}, n, t \mid \boldsymbol{y}, m, t^{\prime}\right)+\mathrm{O}\left(\tau^{2}\right) .
\end{aligned}
$$

Taking the limit $\tau \rightarrow 0$ gives the forward Fokker-Planck equation

$$
\begin{aligned}
& \frac{\partial p\left(\boldsymbol{x}, n, t \mid \boldsymbol{y}, m, t^{\prime}\right)}{\partial t}=\sum_{i=1}^{N} k_{i n} p\left(\boldsymbol{x}, i, t \mid \boldsymbol{y}, m, t^{\prime}\right) \\
& -k_{n+p} p\left(\boldsymbol{x}, n, t \mid \boldsymbol{y}, m, t^{\prime}\right)+D_{n} \Delta_{x} p\left(\boldsymbol{x}, n, t \mid \boldsymbol{y}, m, t^{\prime}\right),
\end{aligned}
$$

with initial condition

$$
p\left(\boldsymbol{x}, n, t^{\prime} \mid \boldsymbol{y}, m, t^{\prime}\right)=\delta_{n m} \delta(\boldsymbol{x}-\boldsymbol{y}) .
$$

To derive the backward equation we start from the relation $\left(t>t^{\prime}+\tau>t^{\prime}\right)$

$$
\begin{gathered}
p\left(\boldsymbol{x}, n, t \mid \boldsymbol{y}, m, t^{\prime}\right)=\int \mathrm{d} \boldsymbol{z} \sum_{i=1}^{N} p\left(\boldsymbol{x}, n, t \mid \boldsymbol{z}, i, t^{\prime}+\tau\right) \\
\times p\left(\boldsymbol{z}, i, t^{\prime}+\tau \mid \boldsymbol{y}, m, t^{\prime}\right),
\end{gathered}
$$

and, by inserting equation (2) and taking the limit $\tau \rightarrow 0$, we obtain the backward Fokker-Planck equation

$$
\begin{gathered}
\frac{\partial p\left(\boldsymbol{x}, n, t \mid \boldsymbol{y}, m, t^{\prime}\right)}{\partial t^{\prime}}=-\left(D_{m} \Delta_{y} p\left(\boldsymbol{x}, n, t \mid \boldsymbol{y}, m, t^{\prime}\right)\right. \\
\left.-\sum_{i=1}^{N} k_{m i}\left(p\left(\boldsymbol{x}, n, t \mid \boldsymbol{y}, m, t^{\prime}\right)-p\left(\boldsymbol{x}, n, t \mid \boldsymbol{y}, i, t^{\prime}\right)\right)\right) .
\end{gathered}
$$

For a particle initially at time $t^{\prime}=0$ in state $m$ at position $\boldsymbol{y}$, the mean sojourn time $u_{n}(\boldsymbol{y}, m)$ it spends in state $n$ is

$$
u_{n}(\boldsymbol{y}, m)=\int_{\Omega} \mathrm{d} \boldsymbol{x} \int_{0}^{\infty} \mathrm{d} t p(\boldsymbol{x}, n, t \mid \boldsymbol{y}, m, 0) .
$$

Because the transition probability $p\left(\boldsymbol{x}, n, t \mid \boldsymbol{y}, m, t^{\prime}\right)$ depends only on $t-t^{\prime}$, by integrating the backward equation we obtain for $u_{n}(\boldsymbol{y}, m)$ the coupled system of equations

$$
D_{m} \Delta u_{n}(\boldsymbol{y}, m)-\sum_{i=1}^{N} k_{m i}\left(u_{n}(\boldsymbol{y}, m)-u_{n}(\boldsymbol{y}, i)\right)=-\delta_{n m},
$$

where the boundary conditions depend on the initial state $m$. For each value $n=1, \ldots, N$, equation (7) constitutes a closed system of equations that can be solved independently. However, it is sufficient to solve the equations only for a specific value of $n$, e.g. $n=1$, because the solutions $u_{n}(\boldsymbol{y}, m)$ for $n>1$ can be obtained from the solutions $u_{1}(\boldsymbol{y}, m)$ by linear transformations. By averaging $u_{n}(\boldsymbol{y}, m)$ over a uniform spatial distribution we obtain the mean sojourn times

$$
u_{n}(m)=\frac{1}{|\Omega|} \int_{\Omega} u_{n}(\boldsymbol{y}, m) \mathrm{d} \boldsymbol{y} .
$$

Finally, from equation (7) we find that the exit times $u(\boldsymbol{y}, m)=$ $\sum_{n=1}^{N} u_{n}(\boldsymbol{y}, m)$ satisfy the coupled system of the mean first passage time equations (see also [47])

$$
D_{m} \Delta u(\boldsymbol{y}, m)-\sum_{i=1}^{N} k_{m i}(u(\boldsymbol{y}, m)-u(\boldsymbol{y}, i))=-1 .
$$

To conclude this part, we emphasize that to derive the sojourn time in state $n$, conditioned on starting in a specific state $m$, one has to consider the sojourn times in state $n$ conditioned on starting in any other state.

\section{Gated narrow escape with exit only in state 1}

From now on we will study the sojourn time equations for a diffusing ligand switching between two states 1,2 and with rates $k_{12}, k_{21}$ and diffusion constants $D_{1}, D_{2}$ (see figure 1). To estimate the mean sojourn times the particle spends in the two states before hitting the absorbing surface patch $\partial \Omega_{\mathrm{a}}$, we analyse equation (7) for $u_{1}(\boldsymbol{x}, 1)$ and $u_{1}(\boldsymbol{x}, 2)$, given by

$$
\begin{gathered}
D_{1} \Delta u_{1}(\boldsymbol{x}, 1)-k_{12}\left(u_{1}(\boldsymbol{x}, 1)-u_{1}(\boldsymbol{x}, 2)\right)=-1 \\
D_{2} \Delta u_{1}(\boldsymbol{x}, 2)-k_{21}\left(u_{1}(\boldsymbol{x}, 2)-u_{1}(\boldsymbol{x}, 1)\right)=0,
\end{gathered}
$$

and $u_{2}(\boldsymbol{x}, 1)$ and $u_{2}(\boldsymbol{x}, 2)$ satisfy equations that are obtained by interchanging $1 \leftrightarrow 2$ in (10). We will first study these equations when the ligand is absorbed at $\partial \Omega_{\mathrm{a}}$ in state 1 while it is reflected everywhere on the boundary in state 2 , and afterwards consider a ligand that is absorbed at $\partial \Omega_{\mathrm{a}}$ in both states.

For a switching ligand absorbed at $\partial \Omega_{\mathrm{a}}$ in state 1 and reflected in state 2 the boundary conditions for equation (10) are

$$
\begin{array}{ll}
u_{1}(\boldsymbol{x}, 1)=0, & \boldsymbol{x} \in \partial \Omega_{\mathrm{a}}, \\
\frac{\partial u_{1}(\boldsymbol{x}, 1)}{\partial n}=0, & \boldsymbol{x} \in \partial \Omega_{\mathrm{r}}, \\
\frac{\partial u_{1}(\boldsymbol{x}, 2)}{\partial n}=0, & \boldsymbol{x} \in \partial \Omega .
\end{array}
$$

The sojourn times $u_{2}(\boldsymbol{x}, 1)$ and $u_{2}(\boldsymbol{x}, 2)$ are obtained from $u_{1}(\boldsymbol{x}, 1)$ and $u_{1}(\boldsymbol{x}, 2)$ through the linear transformation

$$
\left(\begin{array}{l}
u_{2}(\boldsymbol{x}, 1) \\
u_{2}(\boldsymbol{x}, 2)
\end{array}\right)=\frac{k_{12}}{k_{21}}\left(\begin{array}{ll}
1 & 0 \\
0 & 1
\end{array}\right)\left(\begin{array}{l}
u_{1}(\boldsymbol{x}, 1) \\
u_{1}(\boldsymbol{x}, 2)
\end{array}\right)+\left(\begin{array}{c}
0 \\
\frac{1}{k_{21}}
\end{array}\right) .
$$




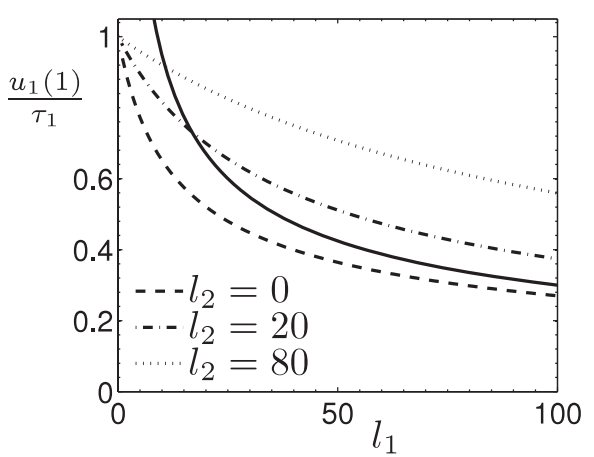

(a)

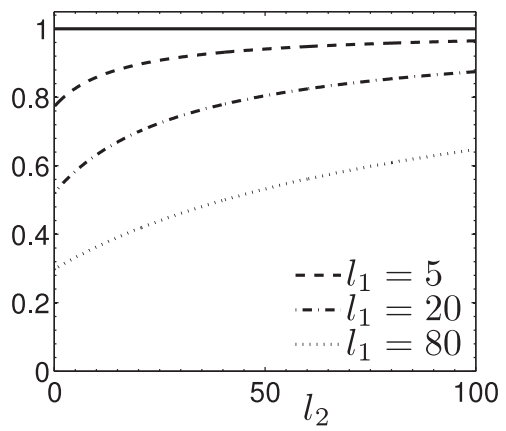

(b)

Figure 2. The sojourn time $u_{1}(1)$ obtained from equation (14) as a function of $l_{1}$ and $l_{2}$, and scaled by the mean first passage time $\tau_{1}$. In panel (a), the continuous curve is the asymptotic limit $3 / \sqrt{l_{1}}$ for $l_{1} \gg 1$ and $\sqrt{l_{1}} \gg l_{2}$, and in panel (b), the continuous curve corresponds to the non-switching case $l_{1}=0$.

From equations (10) and (11) we find that the spatially averaged sojourn times defined in equation (8) satisfy the intuitive relationships

$$
\begin{gathered}
u_{1}(2)=u_{1}(1), \quad u_{2}(1)=u_{1}(1) \frac{k_{12}}{k_{21}}, \\
u_{2}(2)=u_{2}(1)+\frac{1}{k_{21}} .
\end{gathered}
$$

From these relations it follows that the mean times $u(1)$, $u(2)$ and $u$ to exit the domain starting respectively uniformly distributed in state 1 or 2 , and in state 1 and 2 with equilibrium probability $\left(p_{1}, p_{2}\right)=\left(\frac{k_{21}}{k_{12}+k_{21}}, \frac{k_{12}}{k_{12}+k_{21}}\right)$, are

$$
\begin{gathered}
u(1)=u_{1}(1)+u_{2}(1)=u_{1}(1)\left(1+\frac{k_{12}}{k_{21}}\right) \\
u(2)=u_{2}(2)+u_{1}(2)=u(1)+\frac{1}{k_{21}}, \\
u=p_{1} u(1)+p_{2} u(2)=u(1)+\frac{p_{2}}{k_{21}} .
\end{gathered}
$$

In the next sections we first study equation (10) in dimension one, and then in dimension three. In dimension one the equations are integrable and we obtain a full analytical solution, which is very informative because it reveals prominent features that also apply to the three-dimensional case, where only asymptotic formulae are available. We will restrict our discussion to $u(1)$ and $u_{1}(1)$, the discussion for $u(2)$ and $u$ is very similar.

\subsection{Exit time in dimension one}

When $\Omega=(0, L)$ is the one-dimensional interval with an absorbing boundary at $x=0$ in state 1 and reflecting boundary at $x=L$, we obtain in the appendix for $u_{1}(1)$ the formula (see equation (A.11))

$$
u_{1}(1)=\tau_{1}-\frac{l_{1}}{l_{1}+l_{2}}\left(\tau_{1}-\frac{L^{2}}{D_{1}} f\left(l_{1}+l_{2}\right)\right),
$$

where $l_{1}=\frac{k_{12} L^{2}}{D_{1}}, l_{2}=\frac{k_{21} L^{2}}{D_{2}}, \tau_{1}=\frac{L^{2}}{3 D_{1}}$ is the MFPT in state 1 without switching, and $f(x)=\frac{\operatorname{coth} \sqrt{x}}{\sqrt{x}}-\frac{1}{x}$. Apart from the overall scaling factor $L^{2} / D_{1}$, equation (14) shows that $u_{1}(1)$ depends only on the effective parameters $l_{1}$ and $l_{2}$. The asymptotic for $u_{1}(1)$ is

$$
u_{1}(1)= \begin{cases}\frac{L^{2}}{D_{1}} f\left(l_{1}\right)+\mathrm{O}\left(\frac{l_{2}}{l_{1}}\right), & l_{2} \ll l_{1} \\ \tau_{1}+\mathrm{O}\left(\frac{l_{1}}{l_{2}}\right), & l_{2} \gg l_{1},\end{cases}
$$

and for $l_{1} \gg 1$ and $l_{2} \ll \sqrt{l_{1}}$ we have

$$
u_{1}(1) \approx \frac{L^{2}}{D_{1}} \frac{1}{\sqrt{l_{1}}}=\frac{L}{\sqrt{k_{12} D_{1}}}, \quad l_{1} \gg 1, \quad \sqrt{l_{1}} \gg l_{2} .
$$

In figure 2 , we display $u_{1}(1)$ as a function of $l_{1}$ and $l_{2}$. Interestingly, we find the counterintuitive result that the sojourn time $u_{1}(1)$ is always smaller than the mean first passage time $\tau_{1}$ to exit in state 1 without switching. In addition, when the parameters $D_{1}, D_{2}$, and $k_{21}$ are fixed, equation (16) shows that $u_{1}(1)$ becomes arbitrarily small as $k_{12}$ increases. This nonintuitive behaviour deserves more attention and we understand it as follows (see also [36]): for a ligand starting uniformly distributed in state 1 , the probability to be in the neighbourhood of the absorbing boundary at $x=0$ decreases quickly as a function of time. However, after switching to state 2, the distribution is re-homogenized and later on, after switching back from state 2 to 1 , the probability density around $x=0$ is higher compared to the non-switching case. After returning to state 1 , the ligand starts in probability 'closer' to $x=0$ and thus it exits in state 1 faster compared to the non-switching case.

Considering that the sojourn time $u_{1}(1)$ can be arbitrary small, we now explore how this affects the mean time $u(1)$ to exit starting initially in state 1 . From equations (13) and (14) we obtain $\left(\kappa=D_{1} / D_{2}\right)$

$$
\begin{aligned}
& u(1)=u_{1}(1)\left(1+\kappa \frac{l_{1}}{l_{2}}\right) \\
& =\tau_{1}+\frac{l_{1}}{l_{2}}\left(\frac{L^{2}}{D_{1}} f\left(l_{1}+l_{2}\right)+(\kappa-1) u_{1}(1)\right),
\end{aligned}
$$

showing that $u(1) \geqslant \tau_{1}$ for $\kappa \geqslant 1$ : the exit time cannot decrease by switching to a state with a smaller diffusion 


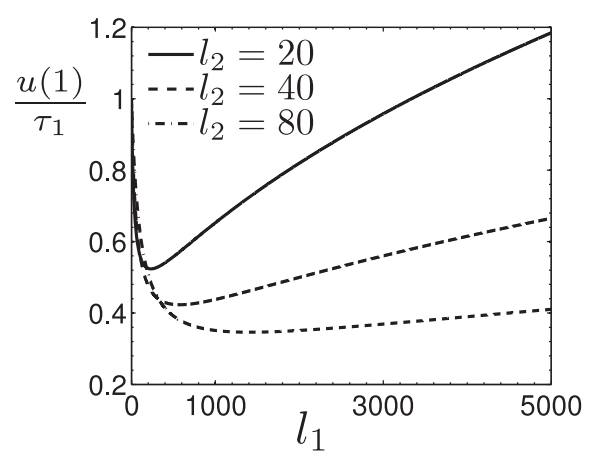

(a)

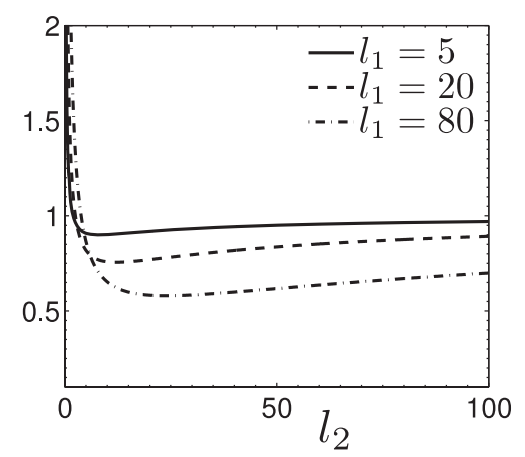

(b)

Figure 3. The mean exit time $u(1)$ obtained from equation (17) as a function of $l_{1}$ and $l_{2}$ for $\kappa=D_{1} / D_{2}=0.1$, and scaled by $\tau_{1}$. The graph of $u(1)$ initially decays steeply, while around and after the minimum it is quite flat. This strong initial dependency on the switching rates may be a way to modulate the signalling time in biochemical systems.

constant. However, as we will show now, this is no longer the case for $\kappa<1$, where one can find switching rates such that $u(1)<\tau_{1}$. Furthermore, if one switching rate is fixed while the other is variable, the graph of $u(1)$ as a function of the variable rate has a minimum with a value that is smaller than $\tau_{1}$. Indeed, using equation (17) for a given value of $l_{2}$, the small $l_{1}$ expansion of $u(1)$ is

$$
u(1)=\tau_{1}+\frac{l_{1}}{l_{2}}\left(\frac{L^{2}}{D_{1}} f\left(l_{2}\right)+(\kappa-1) \tau_{1}\right)+\mathrm{O}\left(l_{1}^{2}\right),
$$

showing that the initial slope $\frac{\partial u}{\partial l_{1}}(1)$ is negative for $l_{2}>\tilde{l}_{2}(\kappa)$, where $\tilde{l}_{2}(\kappa)$ is the root of $f\left(l_{2}\right)+(\kappa-1) / 3=0$. Together with the increasing behaviour $u(1) \sim \sqrt{l_{1}}$ for $l_{1} \rightarrow \infty$, we conclude that $u(1)$ has a minimum smaller than $\tau_{1}$ as function of $l_{1}$ for $l_{2}>\tilde{l}_{2}(\kappa)$. In figure 3(a) we present $u(1)$ as a function of $l_{1}$ for various fixed values of $l_{2}$. In cellular systems, this may correspond to a situation where the diffusion constants and the backward rate $k_{21}$ are determined, but the forward binding rate $k_{12}$ can be adjusted by changing the concentration of a reactant partner. In figure 3(b) we plot $u(1)$ as a function of $l_{2}$ for given $l_{1}$ showing that $u(1)$ also exhibits a minimum in this case.

From figure 3 we observe that the minimum $u(1)_{\mathrm{m}}$ decreases as switching becomes faster. To determine the lower bound for $u(1)_{m}$, we first consider the situation in figure 3(a) where $l_{2}$ and $\kappa$ are fixed and $u_{1}$ is a function of $l_{1}$. The position $l_{1}=l_{1, \mathrm{~m}}\left(l_{2}, \kappa\right)$ of the minimum is found from $\frac{\partial u(1)}{\partial l_{1}}(1)=0$, which leads to the equation (we use equation (17))

$$
-l_{1} \frac{\partial u_{1}(1)}{\partial l_{1}}\left(1+\frac{l_{2}}{l_{1} \kappa}\right)=u_{1}(1) \text {. }
$$

Using the asymptotic of $u_{1}(1)$ for large $l_{1}+l_{2}$, given by $(\hat{\tau}=1 / 3)$

$$
\begin{aligned}
& u_{1}(1)=\frac{L^{2}}{D_{1}}\left(\frac{l_{2}}{l_{1}+l_{2}} \hat{\tau}+\frac{l_{1}}{\left(l_{1}+l_{2}\right) \sqrt{l_{1}+l_{2}}}\right. \\
&\left.+\mathrm{o}\left(\frac{1}{\left(l_{1}+l_{2}\right) \sqrt{l_{1}+l_{2}}}\right)\right),
\end{aligned}
$$

we obtain from equation (18) that the position $\left(l_{1, \mathrm{~m}}, l_{2}\right)$ of the minimum for $l_{2}$ large is determined by

$$
l_{1, \mathrm{~m}}^{3 / 2}=\frac{2 \hat{\tau}(1-\kappa)}{\kappa} l_{2}^{2} .
$$

When $l_{2}$ varies for fixed $l_{1}$ and $\kappa$ (corresponding to the situation depicted in figure $3(\mathrm{~b}))$, the minimum $\left(l_{1}, l_{2, \mathrm{~m}}\right)$ is determined by $\frac{\partial u(1)}{\partial l_{2}}=0$, which for large $l_{1}$ leads to

$$
l_{2, \mathrm{~m}}^{2}=\frac{\kappa}{\hat{\tau}(1-\kappa)} l_{1}^{3 / 2} .
$$

Comparing equation (20) with equation (21) shows that the positions $\left(l_{1, \mathrm{~m}}, l_{2}\right)$ and $\left(l_{1}, l_{2, \mathrm{~m}}\right)$ are different, and thus, $u(1)$ does not have a local or global minimum, which would require that $\left(l_{1, \mathrm{~m}}, l_{2}\right)=\left(l_{1}, l_{2, \mathrm{~m}}\right)$. These results and the scaling laws reflected in equations (20) and (21) share some similarities with [40], where switching occurs between a diffusive and a ballistic motion without orientational control. However, our results differ from a ballistic motion with orientational control, where a global minimum is obtained [43, 47]. Using equation (20) (or equation (21)) we obtain for large switching rates the asymptotic equations

$$
\begin{gathered}
u_{1}(1)_{\mathrm{m}}=\mathrm{O}\left(l_{2}^{-1 / 3}\right), \quad u_{2}(1)_{\mathrm{m}}=\tau_{2}+\mathrm{O}\left(l_{2}^{-1 / 3}\right), \\
u(1)_{\mathrm{m}}=\tau_{2}+\mathrm{O}\left(l_{2}^{-1 / 3}\right)
\end{gathered}
$$

where $\tau_{2}=\kappa \tau_{1}$. Consequently, the lower bound for the exit time $u(1)$ is given by $\tau_{2}$, which is the mean first passage time for a ligand diffusing all the time with the larger diffusion constant $D_{2}$. Surprisingly, but consistent, as $u(1)$ approaches $\tau_{2}$, the ligand spends all the time in state 2, where it cannot exit. Figure 4(a) shows the minimum $u_{1, m}$ scaled by $\tau_{1}$ as a function of $l_{2}$ for various $\kappa$, and confirms the asymptotic relation in equation (20) and the asymptotic behaviour $u_{1, m} \rightarrow$ $\tau_{2}$ for large $l_{2}$. Figure 4(b) displays the corresponding sojourn times $u_{1}(1)_{\mathrm{m}} / \tau_{1}$ and $u_{2}(2)_{\mathrm{m}} / \tau_{2}$ as a function of $l_{2}$ for different values of $\kappa$, showing that $u_{1}(1)_{\mathrm{m}} \rightarrow 0$ and $u_{2}(2)_{\mathrm{m}} \rightarrow \tau_{2}$ as $u(1)_{\mathrm{m}} \rightarrow \tau_{2}$. For $\kappa<1$ exit is fastest if the ligand spends all the time in state 2 , while for $\kappa>1$ exit is fastest if the ligand spends all the time in state 1 , and thus a limit situation occurs for $\kappa=1$. For $\kappa=1$ the ligand exits in a minimal time $u_{1}=\tau_{1}$ by remaining in state 1 , however, there is an additional strategy to exit fast while spending most of the time hidden in the state 2: the strategy consists in choosing $l_{1}$ as large as possible and $l_{2}=\alpha l_{1}$, with $\alpha \ll 1$. We have $u(1) \approx \tau_{1}$ for $\alpha \sqrt{l_{1}} \gg 1$, and 


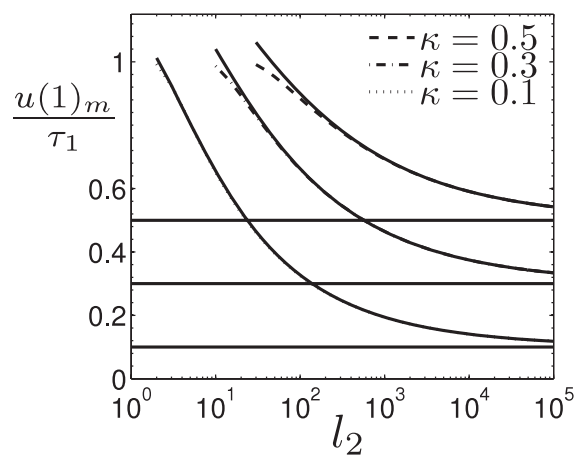

(a)

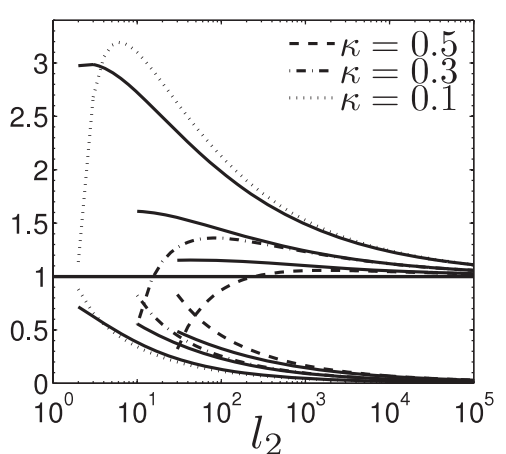

(b)

Figure 4. Panel (a) shows the minimum $u(1)_{m}$ (scaled by $\tau_{1}$ ) of $u(1)$, plotted as a function of $l_{2}$ for various $\kappa$. The horizontal lines display the values of $\kappa$ and the continuous curves are calculated using equation (20). The graphs do not extend up to $l_{2}=0$ because a minimum exists only for $l_{2}>\tilde{l}_{2}(\kappa)$ (see text). Panel (b) shows the ratio $u_{1}(1)_{\mathrm{m}} / \tau_{1}$ (curves below the horizontal line) and $u_{2}(1)_{\mathrm{m}} / \tau_{2}$ (curves above) of the sojourn times. The continuous curves are calculated using equation (20).

since $u_{1}(1) / u_{2}(1)=\alpha$, it follows that the ligand spends only a small fraction $\alpha /(1+\alpha)$ of the time in state 1 .

In summary, we have found that the lower limit of $u(1)$ corresponds to a ligand diffusing all the time with maximal diffusion constant, and interestingly, for $\kappa \leqslant 1$, this implies that fast exit is achieved by diffusing most of the time in state 2 , where exit is not possible. The exit time $u(1)$ has no global or even local minima for $\kappa<1$, and the best strategy to exit fast needs to be adapted to the given constraints. For example, when $k_{21}$ is the unbinding and $k_{12}$ the forward binding rate, then $k_{21}$ usually depends on the local interaction potential, while $k_{12}$ can be modulated by changing the concentration of the binding partner. Furthermore, as shown in figure 3, the graph of $u(1)$ around and past the minimum is quite flat, and thus increasing switching to attain the minimum may not be necessary, because a similar effect can already by achieved at much slower rates. Moreover, because the graph of $u$ (1) decays steeply for small values $l_{1}$ and $l_{2}$, this behaviour provides an efficient mechanism to modulate the activation time, and thus cellular signalling.

\subsection{Gated narrow escape in dimension three}

We now study a switching ligand that can exit a general and regular three-dimensional domain $\Omega$ through a small hole $\partial \Omega_{\mathrm{a}}$ only in state 1 . Without switching, the mean exit time in state 1 equals the NET $\tau_{1} \sim \frac{|\Omega|}{4 a D_{1}}$, where $a$ is the length scale characterizing the small hole $\partial \Omega_{\mathrm{a}}[15,16,18,21]$. Using the dimensionless parameters and functions [21]

$$
\begin{array}{rlrl}
\hat{\boldsymbol{x}}=\frac{\boldsymbol{x}}{a}, & v_{1}(\hat{\boldsymbol{x}}) & =\frac{a D_{1}}{|\Omega|} u_{1}(\boldsymbol{x}, 1), \\
v_{2}(\hat{\boldsymbol{x}})=\frac{a D_{1}}{|\Omega|} u_{1}(\boldsymbol{x}, 2), & l_{1}=\frac{k_{12} a^{2}}{D_{1}}, \quad l_{2}=\frac{k_{21} a^{2}}{D_{2}},
\end{array}
$$

equation (10) are scaled to

$$
\begin{gathered}
\Delta v_{1}(\hat{\boldsymbol{x}})-l_{1}\left(v_{1}(\hat{\boldsymbol{x}})-v_{2}(\hat{\boldsymbol{x}})\right)=-|\hat{\Omega}|^{-1} \\
\Delta v_{2}(\hat{\boldsymbol{x}})+l_{2}\left(v_{1}(\hat{\boldsymbol{x}})-v_{2}(\hat{\boldsymbol{x}})\right)=0,
\end{gathered}
$$

with the boundary conditions

$$
\begin{aligned}
v_{1}(\hat{\boldsymbol{x}})=\frac{\partial v_{2}(\hat{\boldsymbol{x}})}{\partial n}=0, & \hat{\boldsymbol{x}} \in \partial \hat{\Omega}_{\mathrm{a}} \\
\frac{\partial v_{1}(\hat{\boldsymbol{x}})}{\partial n}=\frac{\partial v_{2}(\hat{\boldsymbol{x}})}{\partial n}=0, & \hat{\boldsymbol{x}} \in \partial \hat{\Omega}_{\mathrm{r}}
\end{aligned}
$$

Integrating equation (25) over the scaled domain $\hat{\Omega}$ gives for the spatial averages

$$
v_{1}=\frac{1}{|\hat{\Omega}|} \int_{\hat{\Omega}} v_{1}(\hat{\boldsymbol{x}}) \mathrm{d} \hat{\boldsymbol{x}}=\frac{1}{|\hat{\Omega}|} \int_{\hat{\Omega}} v_{2}(\hat{\boldsymbol{x}}) \mathrm{d} \hat{\boldsymbol{x}}=v_{2} .
$$

Because exact solutions of equations (24) and (25) are not available, we will study the effect of switching by deriving an asymptotic expression for the mean sojourn time $u_{1}(1)=$ $\frac{|\Omega|}{a D_{1}} v_{1}$ in various regimes of the two-dimensional parameter space $\left(l_{1}, l_{2}\right)$. We will explore, in particular, the four regions $l_{1} \ll 1, l_{2}=0$ and $l_{2} \gg l_{1}, l_{2} \ll l_{1}$, and finally $l_{1} \gg l, l_{1} \gg l_{2}$.

3.2.1. The sojourn time $u_{1}(1)$ for $l_{1} \ll 1$. In the regime $l_{1} \ll 1$ we use the regular expansions

$$
\begin{aligned}
& v_{1}(\hat{\boldsymbol{x}})=v_{1}^{0}(\hat{\boldsymbol{x}})+l_{1} v_{1}^{1}(\boldsymbol{x})+l_{1}^{2} v_{1}^{2}(\boldsymbol{x})+\cdots \\
& v_{2}(\hat{\boldsymbol{x}})=v_{2}^{0}(\hat{\boldsymbol{x}})+l_{1} v_{2}^{1}(\boldsymbol{x})+l_{1}^{2} v_{2}^{2}(\boldsymbol{x})+\cdots
\end{aligned}
$$

to study equations (24) and (25). We obtain the sequence of equations

$$
\begin{aligned}
& \Delta\left(v_{1}^{0}(\hat{\boldsymbol{x}})+l_{1} v_{1}^{1}(\boldsymbol{x})\right)-l_{1}\left(v_{1}^{0}(\hat{\boldsymbol{x}})-v_{2}^{0}(\hat{\boldsymbol{x}})\right)+\mathrm{O}\left(l_{1}^{2}\right)=-\frac{1}{|\hat{\Omega}|} \\
& \Delta\left(v_{2}^{0}(\hat{\boldsymbol{x}})+l_{1} v_{2}^{1}(\boldsymbol{x})\right)+l_{2}\left(v_{1}^{0}(\hat{\boldsymbol{x}})-v_{2}^{0}(\hat{\boldsymbol{x}})\right)+l_{1} l_{2}\left(v_{1}^{1}(\hat{\boldsymbol{x}})\right. \\
& \left.\quad-v_{2}^{1}(\hat{\boldsymbol{x}})\right)+\mathrm{O}\left(l_{1}^{2}\right)=0 .
\end{aligned}
$$

and in leading order

$$
\begin{gathered}
\Delta v_{1}^{0}(\hat{\boldsymbol{x}})=-\frac{1}{|\hat{\Omega}|} \\
\Delta v_{2}^{0}(\hat{\boldsymbol{x}})-l_{2} v_{2}^{0}(\hat{\boldsymbol{x}})=-l_{2} v_{1}^{0}(\hat{\boldsymbol{x}}) .
\end{gathered}
$$


The leading order $v_{1}^{0}(\hat{\boldsymbol{x}})$ satisfies the usual NET equation with solution $v_{1}^{0}(\hat{\boldsymbol{x}})=\hat{\tau}(\hat{\boldsymbol{x}})$, where the NET function $\hat{\tau}(\hat{\boldsymbol{x}})$ is well approximated by its spatial average $\hat{\tau} \sim \mathrm{O}(1)$ outside a boundary layer of the absorbing window (in scaled coordinates the radius of the boundary layer is of order 1) $[6,13,15,16,19-21]$. The leading order term is $v_{2}^{0}(\hat{\boldsymbol{x}})$ and depends on $l_{2}$. For $l_{2} \ll 1$, we approximate

$$
v_{2}^{0}(\hat{\boldsymbol{x}}) \approx v_{1}^{0}=\hat{\tau}
$$

and in the limit $l_{2} \gg 1$, we have outside a boundary layer of the absorbing window

$$
v_{2}^{0}(\hat{\boldsymbol{x}})=\left(1-\frac{1}{l_{2}} \Delta\right)^{-1} v_{1}^{0}(\hat{\boldsymbol{x}}) \approx v_{1}^{0}(\hat{\boldsymbol{x}})+\mathrm{O}\left(\frac{1}{l_{2}}\right) .
$$

Because the first order correction $v_{1}^{1}(\hat{\boldsymbol{x}})$ is solution of $\Delta v_{1}^{1}(\boldsymbol{x})=v_{1}^{0}(\hat{\boldsymbol{x}})-v_{2}^{0}(\hat{\boldsymbol{x}})$ and is bounded, we obtain for the mean sojourn time

$$
u_{1}(1)=\frac{|\Omega|}{a D_{1}}\left(\hat{\tau}+\mathrm{O}\left(l_{1}\right)\right) \approx \tau_{1}, \quad l_{1} \ll 1
$$

where $\tau_{1}=\frac{|\Omega|}{a D_{1}} \hat{\tau}$ is the NET in state 1 . To obtain a more physical interpretation of the asymptotic results we observe that the effective parameters $l_{1}=k_{12} a^{2} / D_{1}$ and $l_{2}=k_{21} a^{2} / D_{2}$ involve the length $a$, which roughly characterizes the radius of the boundary layer around the small hole. Roughly speaking, $l_{1}$ and $l_{2}$ compare the switching times $1 / k_{21}$ and $1 / k_{21}$ to the times $a^{2} / D_{1}$ and $a^{2} / D_{2}$ that a particle spends in the boundary layer. For example, if $\left(l_{1}, l_{2}\right) \gtrsim 1$, switching is fast enough to affect the motion of the ligand while it diffuses inside the boundary layer, while for $\left(l_{1}, l_{2}\right) \ll 1$ switching is too slow. From these observations we obtain the following interpretation: for $l_{1} \ll 1$, switching from state 1 to 2 is too slow to affect the behaviour of the particle in state 1 while it is inside the boundary layer, and the sojourn time $u_{1}(1)$ is not changed compared to the NET $\tau_{1}$. For $l_{2} \ll 1$, the ligand in state 2 explores a domain much larger than the boundary layer, and thus the positional sojourn time $u_{1}(\boldsymbol{x}, 2)$ is almost constant. For $l_{2} \ll 1$, the ligand switches very fast from state 2 to state 1 , and thus $u_{1}(\boldsymbol{x}, 2) \approx u_{1}(\boldsymbol{x}, 1)$.

3.2.2. The sojourn time $u_{1}$ (1) for $l_{2}=0$. The condition $l_{2}=$ 0 corresponds to the limit $D_{2} \rightarrow \infty$ and not $k_{21} \rightarrow 0$, since the latter does not have a finite time $u_{1}(\boldsymbol{x}, 2)$. The physical situation corresponds to a ligand that becomes uniformly distributed in $\Omega$ after switching to state 2 . The solution of equation (25) for $l_{2}=0$ is $v_{2}(\hat{\boldsymbol{x}})=v_{1}=$ const, which is intuitive because fast diffusion in state 2 wipes out any initial position dependency. From equation (24) we obtain for $v_{1}(\hat{\boldsymbol{x}})$ the equation

$$
\Delta v_{1}(\hat{\boldsymbol{x}})-l_{1} v_{1}(\hat{\boldsymbol{x}})=-\left(\frac{1}{|\hat{\Omega}|}+l_{1} v_{1}\right)
$$

Thus, $v_{1}(\hat{\boldsymbol{x}})$ satisfies the narrow escape equation with a killing rate $l_{1}$ and an additional source term $l_{1} v_{1}$, which ensures that the particle is not killed by switching to state 2 , but reappears uniformly redistributed in state 1 , and it can only exit through the absorbing hole.

For $l_{1} \ll 1$, the analysis of equation (31) is similar to section 3.2.1 and gives in leading order $u_{1}(1) \approx \tau_{1}$. We shall now focus on $l_{1} \gg 1$. Because the ligand becomes uniformly distributed in state 2 , the sojourn time $u_{1}(\boldsymbol{x}, 1)$ depends on the initial position $\boldsymbol{x}$ only if the distance to the absorbing hole is small enough that the particle has a significant probability to exit before switching. From this we conclude that the boundary layer of $u_{1}(\boldsymbol{x}, 1)$ (resp. $\left.v_{1}(\hat{\boldsymbol{x}})\right)$ necessarily shrinks to zero as $l_{1} \rightarrow \infty$. To estimate $v_{1}(\hat{\boldsymbol{x}})$ near $\partial \hat{\Omega}_{\mathrm{a}}$ for $l_{1} \gg 1$, we use boundary layer analysis: because the boundary is regular, in a local and orthogonal coordinate frame $\left(\rho, \hat{\boldsymbol{x}}_{t}\right)$ centred at the origin of the absorbing hole $\partial \hat{\Omega}_{\mathrm{a}}$, where $\rho=-\operatorname{dist}(P, \partial \hat{\Omega})$ and $P=\left(\rho, \hat{\boldsymbol{x}}_{t}\right)$, equation (31) becomes

$$
\frac{\partial^{2}}{\partial \rho^{2}} v_{1}\left(\rho, \hat{\boldsymbol{x}}_{t}\right)+\Delta_{\hat{\boldsymbol{x}}_{t}} v_{1}\left(\rho, \hat{\boldsymbol{x}}_{t}\right)-l_{1} v_{1}\left(\rho, \hat{\boldsymbol{x}}_{t}\right)=-\left(\frac{1}{|\hat{\Omega}|}+l_{1} v_{1}\right) \text {. }
$$

Using the separation of variables, we obtain the boundary layer expansion

$$
v_{1}^{\mathrm{BL}}\left(\rho, \hat{\boldsymbol{x}}_{t}\right)=A\left(\hat{\boldsymbol{x}}_{t}\right) \mathrm{e}^{-\sqrt{l_{1}} \rho}+\left(\frac{1}{l_{1}|\hat{\Omega}|}+v_{1}\right),
$$

with $\Delta_{\hat{\boldsymbol{x}}_{t}} A\left(\boldsymbol{x}_{t}\right)=0$. To find $A\left(\hat{\boldsymbol{x}}_{t}\right)$ we use $v_{1}^{\mathrm{BL}}\left(0, \hat{\boldsymbol{x}}_{t}\right)=0$ for $\hat{\boldsymbol{x}}_{t} \in \partial \hat{\Omega}_{\mathrm{a}}$ and get $A\left(\hat{\boldsymbol{x}}_{t}\right)=-\left(\frac{1}{l_{1}|\hat{\Omega}|}+v_{1}\right)$, and thus

$$
v_{1}^{\mathrm{BL}}\left(\rho, \hat{\boldsymbol{x}}_{t}\right) \approx\left(\frac{1}{l_{1}|\hat{\Omega}|}+v_{1}\right)\left(1-\mathrm{e}^{-\sqrt{l_{1}} \rho}\right) .
$$

To determine $v_{1}$, we integrate equation (31) and obtain the flux condition

$$
\begin{aligned}
& -1=\int_{\partial \hat{\Omega}_{\mathrm{a}}} \frac{\partial v_{1}(\hat{\boldsymbol{x}})}{\partial \boldsymbol{n}_{x}} \mathrm{~d} \boldsymbol{S}_{x}=-\int_{\partial \hat{\Omega}_{\mathrm{a}}} \frac{\partial v_{1}^{\mathrm{BL}}\left(0, \boldsymbol{x}_{t}\right)}{\partial \rho} \mathrm{d} S_{x} \\
& \approx\left|\partial \hat{\Omega}_{\mathrm{a}}\right|\left(\frac{1}{l_{1}|\hat{\Omega}|}+v_{1}\right) \sqrt{l_{1}},
\end{aligned}
$$

from which we find

$$
v_{1}=\frac{1}{\left|\partial \hat{\Omega}_{\mathrm{a}}\right| \sqrt{l_{1}}}+\mathrm{O}\left(\frac{1}{l_{1}|\hat{\Omega}|}\right) .
$$

We further remark that for $l_{1} \ll 1$, the local flux through $\partial \hat{\Omega}_{\mathrm{a}}$ is almost position independent, and given by

$$
\frac{\partial v_{1}(\hat{\boldsymbol{x}})}{\partial \boldsymbol{n}_{x}} \approx \frac{1}{\left|\partial \hat{\Omega}_{\mathrm{a}}\right|}
$$

which is in contrast with the usual NET flux $[19,20]$. Finally, the asymptotic result for the sojourn time is

$$
u_{1}(1)=\left\{\begin{array}{ll}
\tau_{1}, & l_{1} \ll 1 \\
\frac{|\Omega|}{a D_{1}\left|\partial \hat{\Omega}_{\mathrm{a}}\right| \sqrt{l_{1}}}=\frac{|\Omega|}{\left|\partial \Omega_{\mathrm{a}}\right| \sqrt{D_{1} k_{12}}}, & l_{1} \gg 1
\end{array} .\right.
$$

Similar to the one-dimensional case (see equation (16)), we find that the sojourn time $u_{1}(1)$ shrinks to zero as $l_{1} \rightarrow \infty$. For a circular hole of radius $a$, using $\tau_{1} \approx \frac{|\Omega|}{4 a D_{1}}$ [6], we get

$$
u_{1}(1) \approx \tau_{1} \frac{4}{\pi \sqrt{l_{1}}}, \quad l_{1} \gg 1 .
$$


3.2.3. The sojourn time $u_{1}(1)$ for $l_{2} \gg l_{1}$. When $l_{2} \gg l_{1}$, we sum equations (24) and (25) to get the equation

$$
\Delta\left(v_{1}(\hat{\boldsymbol{x}})+\xi v_{2}(\hat{\boldsymbol{x}})\right)=-\frac{1}{|\hat{\Omega}|},
$$

where $\xi=\frac{l_{1}}{l_{2}} \ll 1$. Using a regular expansion of $v_{1}(\hat{\boldsymbol{x}})$ and $v_{2}(\hat{\boldsymbol{x}})$ in the small parameter $\xi$ we find in leading order $v_{1}^{0}(\hat{\boldsymbol{x}})=\hat{\tau}(\hat{\boldsymbol{x}})$, which gives

$$
u_{1}(1) \approx \tau_{1} \text {. }
$$

Inserting $v_{1}^{0}(\hat{\boldsymbol{x}})=\hat{\tau}(\hat{\boldsymbol{x}})$ into equation (25) leads back to equation (28) for $v_{2}^{0}(\hat{\boldsymbol{x}})$, which we have already discussed. To give a physical interpretation, for $l_{2} \gg l_{1}$, the ligand in state 2 explores an area that is much smaller than what it would have explored in state 1 without switching, and therefore the excursions to state 2 do not affect its behaviour in state 1 , which leads to $u_{1}(1) \approx \tau_{1}$.

3.2.4. The sojourn time $u_{1}(1)$ for $l_{1} \gg 1$ and $l_{1} \gg l_{2}$. When $l_{1} \gg 1$ and $l_{1} \gg l_{2}$, we now show that the leading order asymptotic for $u_{1}(1)$ is given by equation (37) found for $l_{1} \gg 1, l_{2}=0$. We rewrite equation (39) as

$$
\Delta\left(\eta v_{1}(\hat{\boldsymbol{x}})+v_{2}(\hat{\boldsymbol{x}})\right)=-\frac{\eta}{|\hat{\Omega}|},
$$

where $\eta=\frac{l_{2}}{l_{1}} \ll 1$ and introduce the function $\varphi(\hat{\boldsymbol{x}})$ defined by $v_{2}(\hat{\boldsymbol{x}})=v_{1}+\eta\left(\varphi(\hat{\boldsymbol{x}})-v_{1}(\hat{\boldsymbol{x}})\right)$, which satisfies

$$
\Delta \varphi(\hat{\boldsymbol{x}})=-\frac{1}{|\hat{\Omega}|},
$$

with boundary conditions

$$
\frac{\partial \varphi(\hat{\boldsymbol{x}})}{\partial n}=\frac{\partial v_{1}(\hat{\boldsymbol{x}})}{\partial n}, \quad \hat{\boldsymbol{x}} \in \partial \hat{\Omega}_{\mathrm{a}}
$$

and

$$
\frac{\partial \varphi(\hat{\boldsymbol{x}})}{\partial n}=0, \quad \hat{\boldsymbol{x}} \in \partial \hat{\Omega}_{\mathrm{r}}
$$

Because $v_{1}=v_{2}$, the spatial average of $\varphi(\hat{\boldsymbol{x}})$ is $\varphi=v_{1}$. Inserting $v_{2}(\hat{\boldsymbol{x}})=v_{1}+\eta\left(\varphi(\hat{\boldsymbol{x}})-v_{1}(\hat{\boldsymbol{x}})\right)$ into equation (24) gives

$$
\Delta v_{1}(\hat{\boldsymbol{x}})-l_{1}\left[v_{1}(\hat{\boldsymbol{x}})-v_{1}-\eta\left(\varphi(\hat{\boldsymbol{x}})-v_{1}(\hat{\boldsymbol{x}})\right)\right]=-\frac{1}{|\hat{\Omega}|},
$$

and using the regular expansion of $v_{1}(\hat{\boldsymbol{x}})$ and $\varphi(\hat{\boldsymbol{x}})$ in the small parameter $\eta$ we recover for the leading order term $v_{1}^{0}(\hat{\boldsymbol{x}})$ equation (31). The first order expression for $\varphi^{0}(\hat{\boldsymbol{x}})$ is obtained by using $v_{1}^{0}(\hat{\boldsymbol{x}})$ to evaluate the boundary conditions of equation (42). Because the flux coming from $v_{1}^{0}(\hat{\boldsymbol{x}})$ is of order $1 /\left|\partial \hat{\Omega}_{\mathrm{a}}\right| \sim 1$ (see equation $(36)$ ), $\varphi^{0}(\hat{\boldsymbol{x}})$ is of order 1 , and contrary to $v_{1}^{0}(\hat{\boldsymbol{x}})$, it does not vanish for large $l_{1}$. Finally, from equation (43), $v_{1}^{0}(\hat{\boldsymbol{x}})$ is a good approximation to $v_{1}(\hat{\boldsymbol{x}})$ for $\left|v_{1}^{0}(\hat{\boldsymbol{x}})-v_{1}^{0}\right| \gg \eta\left|\varphi^{0}(\hat{\boldsymbol{x}})-v_{1}^{0}(\hat{\boldsymbol{x}})\right|$. Because $\left|\varphi^{0}(\hat{\boldsymbol{x}})-v_{1}^{0}(\hat{\boldsymbol{x}})\right|$ is of order 1 , while $\left|v_{1}^{0}(\hat{\boldsymbol{x}})-v_{1}^{0}\right|=\mathrm{O}\left(\frac{1}{\sqrt{l_{1}}}\right)$ (see the analysis for $l_{2}=$
0 ), we obtain that $v_{1}^{0}(\hat{\boldsymbol{x}})$ is a good first order approximation for $\sqrt{l_{1}} \gg l_{2}$. Finally, we obtain the expression

$$
\begin{gathered}
u_{1}(1) \approx \frac{|\Omega|}{a D_{1}\left|\partial \hat{\Omega}_{\mathrm{a}}\right| \sqrt{l_{1}}}=\frac{|\Omega|}{\left|\partial \Omega_{\mathrm{a}}\right| \sqrt{D_{1} k_{12}}}, \\
l_{1} \gg 1, \quad \sqrt{l_{1}} \gg l_{2},
\end{gathered}
$$

which also includes the case $l_{2}=0$.

3.2.5. Summary of the asymptotic results for $u_{1}(1)$ and $u(1)$. By collecting all the previous asymptotic results for $u_{1}(1)$ we summarize

$$
u_{1}(1)= \begin{cases}\tau_{1}, \quad l_{1} \ll 1 & \text { or } l_{2} \gg l_{1} \\ \frac{|\Omega|}{\left|\partial \Omega_{\mathrm{a}}\right| \sqrt{D_{1} k_{12}}}, & l_{1} \gg 1 \text { and } \sqrt{l_{1}} \gg l_{2},\end{cases}
$$

and from this we obtain for the GNET $u(1)$ the asymptotic expressions

$$
u(1)=\left(1+\frac{k_{12}}{k_{21}}\right)\left\{\begin{array}{c}
\tau_{1}, \quad l_{1} \ll 1 \text { or } l_{2} \gg l_{1} \\
\frac{|\Omega|}{\left|\partial \Omega_{\mathrm{a}}\right| \sqrt{D_{1} k_{12}}}, \\
l_{1} \gg 1 \text { and } \sqrt{l_{1} \gg l_{2} .}
\end{array}\right.
$$

In figure 5(a) we compare our asymptotic results for $u_{1}$ (1) with simulation of Brownian motion together with the Gillespie algorithm [48] to model switching (the simulations are performed in a sphere of radius $r=30$ with a circular hole of radius $a=1$ ). Figure 5(a) confirms our asymptotic formula given in equation (38), and shows that $u_{1}(1)$ decreases with increasing $l_{1}$. Figure 5(b) displays the simulation results for $u(1)$ as a function of $l_{1}$ for various $l_{2}$ and $\kappa=D_{1} / D_{2}=0.1$. Similar to our findings in dimension one (see figure 3(a)), $u(1)$ has a minimum attained at some value $l_{1}>0$. Furthermore, although we do not have a mathematical proof, it is reasonable to assume that $u(1)$ has the lower bound $\tau_{2}=\kappa \tau_{1}$ attained asymptotically for very fast switching, similar to what we found in dimension one.

Equation (45) shows that the sojourn time $u_{1}(1)$ differs from the NET $\tau_{1}$ only if $l_{1} \gg 1$ such that switching is fast enough to affect the particle motion inside the boundary layer. In the range where $u_{1}(1) \approx \tau_{1}$, the GNET $u(1)$ in equation (46) is the NET $\tau_{1}$ divided by the probability $p_{1}=$ $\frac{k_{21}}{k_{12}+k_{21}}$ to find the ligand in state 1 , which corresponds to a mean-field situation where switching and absorption proceed independently, see also [36]. In this range the switching dynamics can be approximated by a non-switching diffusion process with an effective diffusion constant $D_{\text {eff }}=D_{1} / p_{1}$. In the range $\sqrt{l_{1}} \gg l_{2}, l_{1} \gg 1, u(1)$ is inversely proportional to the surface of the hole, similar to the reaction-controlled NET to a partially absorbing hole $[21,36]$. This expression for $u(1)$ is very different from the mean-field result and indicates strong correlations between switching and absorption. In general, deviations from a mean-field situation occur if switching from state 1 to 2 is fast enough to affect the diffusion of the particle inside the boundary layer, and second, diffusion in state 2 has to be such that the probability distribution inside the boundary layer becomes re-homogenized. 


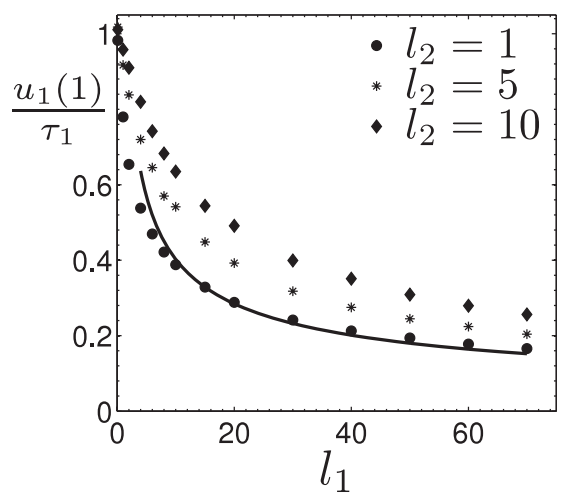

(a)

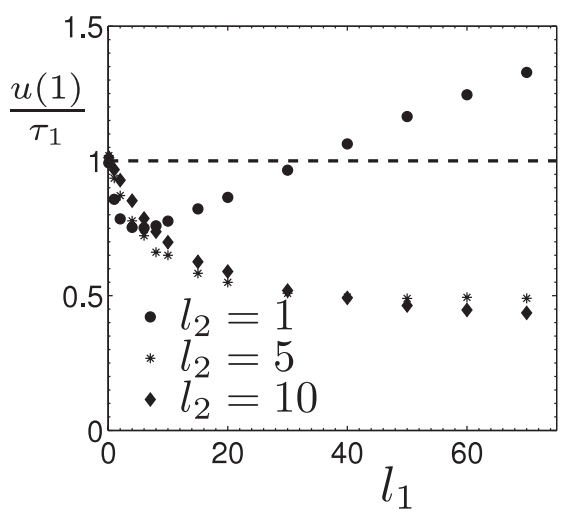

(b)

Figure 5. The ratios $u_{1}(1) / \tau_{1}$ and $u(1) / \tau_{1}$ obtained from Brownian simulations as a function of $l_{1}$ for different values $l_{2}$ (marked by various symbols). The results for $u(1)$ in panel (b) are obtained with $\kappa=0.1$. The Brownian simulations are performed in a sphere of radius $r=30$ with an absorbing circular hole of radius $a=1$. The continuous line in panel (a) is the asymptotic $\frac{4}{\pi \sqrt{l_{1}}}$ (see equation (38)), which is a good approximation for $l_{2} \lesssim 1$ and $l_{1} \geqslant 10$.

\section{Gated narrow escape with exit in both states}

To complete the switching analysis, we now consider a three-dimensional domain where the diffusing ligand can be absorbed at $\partial \Omega_{\mathrm{a}}$ in both states. The boundary conditions

$u_{1}(\boldsymbol{x}, 1)=u_{1}(\boldsymbol{x}, 2)=u_{2}(\boldsymbol{x}, 1)=u_{2}(\boldsymbol{x}, 2)=0$

for $x \in \partial \Omega_{\mathrm{a}}$

$\frac{\partial u_{1}(\boldsymbol{x}, 1)}{\partial n}=\frac{\partial u_{1}(\boldsymbol{x}, 2)}{\partial n}=\frac{\partial u_{2}(\boldsymbol{x}, 1)}{\partial n}=\frac{\partial u_{2}(\boldsymbol{x}, 2)}{\partial n}=0$

for $\boldsymbol{x} \in \partial \Omega_{\mathrm{r}}$,

are identical in both states, and thus the solutions $u_{2}(\boldsymbol{x}, 2)$ and $u_{2}(\boldsymbol{x}, 1)$ are obtained from $u_{1}(\boldsymbol{x}, 1)$ and $u_{1}(\boldsymbol{x}, 2)$ by interchanging $1 \leftrightarrow 2$. By subtracting and adding the two equations in equation (10) we find that $\tau_{1}(\boldsymbol{x})=u_{1}(\boldsymbol{x}, 1)+$ $\frac{l_{1}}{l_{2}} u_{1}(\boldsymbol{x}, 2)$ and $\tilde{\tau}_{1}(\boldsymbol{x})=u_{1}(\boldsymbol{x}, 1)-u_{1}(\boldsymbol{x}, 2)$ satisfy the decoupled system of equations

$$
\begin{gathered}
D_{1} \Delta \tau_{1}(\boldsymbol{x})=-1, \\
D_{1} \Delta \tilde{\tau}_{1}(\boldsymbol{x})-D_{1}\left(l_{1}+l_{2}\right) \tilde{\tau}_{1}(\boldsymbol{x})=-1
\end{gathered}
$$

with boundary conditions

$$
\tau_{1}(x)=\tilde{\tau}_{1}(x)=0, \quad x \in \partial \Omega_{\mathrm{a}}
$$

and

$$
\frac{\partial \tau_{1}(\boldsymbol{x})}{\partial n}=\frac{\partial \tilde{\tau}_{1}(\boldsymbol{x})}{\partial n}=0, \quad \boldsymbol{x} \in \partial \Omega_{\mathrm{r}}
$$

where $l_{1}=\frac{k_{12}}{D_{1}}$ and $l_{2}=\frac{k_{21}}{D_{2}}$. Thus, $\tau_{1}(\boldsymbol{x})$ is the NET for a Brownian ligand diffusing with diffusion constant $D_{1}$, and $\tilde{\tau}_{1}(x)$ is the survival time in $\Omega$ with a uniform killing rate $D_{1}\left(l_{1}+l_{2}\right)$. To estimate $\tilde{\tau}_{1}(\boldsymbol{x})$ for a small killing rate, we approximate the inhomogeneous term in equation (48) by its spatial average and obtain $D_{1} \Delta \tilde{\tau}_{1}(\boldsymbol{x})=-1+D_{1}\left(l_{1}+l_{2}\right) \tilde{\tau}_{1}$, where $\tilde{\tau}_{1}$ is the spatial average of $\tilde{\tau}_{1}(\boldsymbol{x})$. The solution of this equation is $\tilde{\tau}_{1}(\boldsymbol{x})=\left(1-D_{1}\left(l_{1}+l_{2}\right) \tilde{\tau}_{1}\right) \tau_{1}(\boldsymbol{x})$, and by taking the spatial average and solving for $\tilde{\tau}_{1}$ we obtain

$$
\tilde{\tau}_{1}=\frac{\tau_{1}}{1+D_{1}\left(l_{1}+l_{2}\right) \tau_{1}}=\frac{\tau_{1}}{1+k_{12} \tau_{1}+k_{21} \tau_{2}},
$$

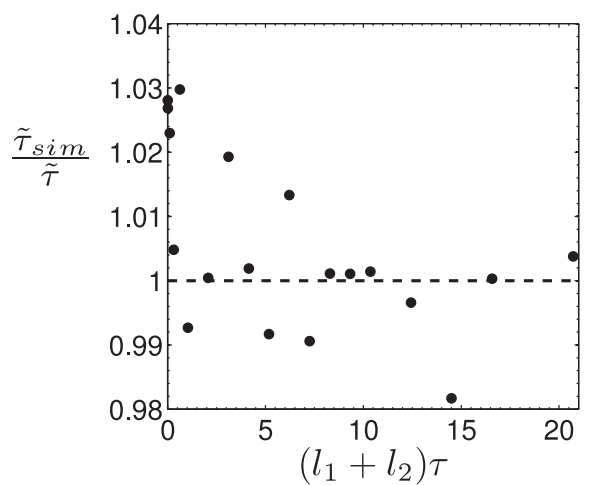

Figure 6. Comparison of the analytical value $\tilde{\tau}$ predicted by equation (49) with $\tilde{\tau}_{\text {sim }}$ obtained from Brownian simulations with 10000 diffusing particles in a sphere of radius $r=30$ with a circular absorbing hole of radius $a=1$ (for the diffusion constant we used $D=1) . \tilde{\tau}$ in equation (49) is calculated using $\tau=\frac{|\Omega|}{4 a}$.

where $\tau_{1}$ is the spatial average of $\tau_{1}(\boldsymbol{x})$ and $\tau_{2}=\frac{D_{1}}{D_{2}} \tau_{1}$. Although equation (49) was derived assuming a small killing rate, interestingly, it also gives the correct asymptotic $\tilde{\tau}_{1}=$ $1 /\left(D_{1}\left(l_{1}+l_{2}\right)\right)$ for a large killing rate. To clarify the validity range of equation (49), we compare in figure 6 this expression with the results of Brownian simulation, confirming that equation (49) is a valid approximation for arbitrary values $k_{12} \tau_{1}+k_{21} \tau_{2}$.

Finally, expressing $u_{1}(\boldsymbol{x}, 1)$ and $u_{1}(\boldsymbol{x}, 2)$ as functions of $\tau_{1}(\boldsymbol{x})$ and $\tilde{\tau}_{1}(\boldsymbol{x})$, we obtain

$$
u_{1}(\boldsymbol{x}, 1)=\frac{l_{2}}{l_{1}+l_{2}}\left(\tau_{1}(\boldsymbol{x})+\frac{l_{1}}{l_{2}} \tilde{\tau}_{1}(\boldsymbol{x})\right)
$$

$$
u_{1}(\boldsymbol{x}, 2)=\frac{l_{2}}{l_{1}+l_{2}}\left(\tau_{1}(\boldsymbol{x})-\tilde{\tau}_{1}(\boldsymbol{x})\right)=u_{1}(\boldsymbol{x}, 1)-\tilde{\tau}_{1}(\boldsymbol{x}) .
$$

By interchanging $1 \leftrightarrow 2$ we obtain for $u_{2}(\boldsymbol{x}, 1)$ and $u_{2}(\boldsymbol{x}, 2)$ the expressions

$u_{2}(\boldsymbol{x}, 1)=\frac{l_{1}}{l_{1}+l_{2}}\left(\tau_{2}(\boldsymbol{x})-\tilde{\tau}_{2}(\boldsymbol{x})\right)=\frac{k_{12}}{k_{21}} u_{1}(\boldsymbol{x}, 2)$, 


$$
\begin{array}{r}
u_{2}(\boldsymbol{x}, 2)=\frac{l_{1}}{l_{1}+l_{2}}\left(\tau_{2}(\boldsymbol{x})+\frac{l_{2}}{l_{1}} \tilde{\tau}_{2}(\boldsymbol{x})\right) \\
=\frac{D_{1}}{D_{2}}\left(u_{1}(\boldsymbol{x}, 1)-\frac{l_{2}-l_{1}}{l_{2}} u_{1}(\boldsymbol{x}, 2)\right),
\end{array}
$$

where $\tau_{2}(\boldsymbol{x})=\frac{D_{1}}{D_{2}} \tau_{1}(\boldsymbol{x})$ and $\tilde{\tau}_{2}(\boldsymbol{x})=\frac{D_{1}}{D_{2}} \tilde{\tau}_{1}(\boldsymbol{x})$. Thus, the linear transformation that relates $\left(u_{1}(\boldsymbol{x}, 1), u_{1}(\boldsymbol{x}, 2)\right.$ to $\left(u_{2}(\boldsymbol{x}, 1), u_{2}(\boldsymbol{x}, 2)\right)$ is

$$
\left(\begin{array}{l}
u_{2}(\boldsymbol{x}, 1) \\
u_{2}(\boldsymbol{x}, 2)
\end{array}\right)=\frac{D_{1}}{D_{2}}\left(\begin{array}{cc}
0 & \frac{l_{1}}{l_{2}} \\
1 & -\frac{l_{2}-l_{1}}{l_{2}}
\end{array}\right)\left(\begin{array}{l}
u_{1}(\boldsymbol{x}, 1) \\
u_{1}(\boldsymbol{x}, 2)
\end{array}\right)
$$

which has to be compared to the transformation in equation (11) with absorption in state 1 only. Because the boundary conditions in state 1 and 2 are identical, the transformation in equation (54) can also mix solutions with different initial states, which is not possible in equation (11).

Using formula (49) for $\tilde{\tau}_{1}$, the expression for the spatially averaged sojourn times are

$$
\begin{aligned}
& u_{1}(1)=\frac{l_{2}}{l_{1}+l_{2}}\left(\tau_{1}+\frac{l_{1}}{l_{2}} \tilde{\tau}_{1}\right)=\tau_{1} \frac{1+k_{21} \tau_{2}}{1+k_{12} \tau_{1}+k_{21} \tau_{2}}, \\
& u_{1}(2)=\frac{l_{2}}{l_{1}+l_{2}}\left(\tau_{1}-\tilde{\tau}_{1}\right)=\tau_{1} \frac{k_{21} \tau_{2}}{1+k_{12} \tau_{1}+k_{21} \tau_{2}},
\end{aligned}
$$

and $u_{2}(1), u_{2}(2)$ are obtained by interchanging $1 \leftrightarrow 2$. The mean time $u(1)=u_{1}(1)+u_{2}(1)$ to exit the domain starting uniformly distributed in state 1 is

$$
\begin{aligned}
& u(1)=\frac{l_{2} \tau_{1}+l_{1} \tau_{2}}{l_{1}+l_{2}}+\frac{l_{1}}{l_{1}+l_{2}}\left(\tilde{\tau}_{1}-\tilde{\tau}_{2}\right) \\
& =\tau_{1} \frac{1+\left(k_{12}+k_{21}\right) \tau_{2}}{1+k_{12} \tau_{1}+k_{21} \tau_{2}} .
\end{aligned}
$$

Finally, we observe that the switching results when the ligand can exit in both states are not related to any boundary layer effect, contrary to a ligand exiting in state 1 only.

\section{Discussion and conclusion}

We extended here the narrow escape time formulae to a diffusing ligand randomly switching between two states 1 and 2 with diffusion coefficients $D_{1}$ and $D_{2}$ under consideration of the sojourn times the ligand spends in the different states before exiting. Therefore, we did not analyse the mean first passage time equations, but rather the equations for the sojourn times, which give a more accurate picture of the switching dynamics. We obtained new formulae for the GNET when exit is possible only in one state (see equation (46)) or in both states (see equation (57)).

When the ligand can exit only in state 1 , we find unexpected phenomena: first, switching always decreases the sojourn time in state 1 compared to the non-switching case, and the sojourn time can asymptotically go to zero for fast switching rates. Second, fastest exit is achieved by diffusing all the time in the state with the largest diffusion constant. For $D_{1}>D_{2}$ this is not surprising, because the ligand should stay all the time in the state where it can also exit. However, for $D_{2}>D_{1}$ this result is no longer intuitive, because now fastest exit is achieved asymptotically for very fast switching rates, where the rates have to be such that the ligand spends most of the time 'hidden' in state 2, where it cannot exit. We identify the effective parameters $l_{1}$ and $l_{2}$ (see equation (23)) that determine this behaviour, and we show that these phenomena occur if switching is so fast that it effects the behaviour of the ligand in the boundary layer around the absorbing window. Finally, for $D_{2}>D_{1}$, if one switching rate is constrained, one can adapt the other rate such as to minimize the GNET. For example, if switching is due to binding, the backward rate is usually constrained, while the forward rate can be easily adjusted through the concentration of the binding partner. Furthermore, as shown in figures 3 and 5, the behaviour of the GNET around the minimum is quite flat, while it decays steeply for small switching rates. Thus, to efficiently modulate activation time it seems unfavourable to operate near the minimum, but rather in the fast decay region.

We end by sketching two applications in cellular biology where our results for switching between two diffusive states might be relevant. The first application concerns the search time for a promoter DNA-site by a transcription factor (TF), which alternates between a three-dimensional diffusion in the nucleus and one-dimensional diffusion along the DNA [49-51]. During its one-dimensional diffusion along the DNA, in order to speed up diffusion in a rough energy potential, it has been proposed that the TF alternates between two states (conformations) [52-57]: in state 1, the TF has a high affinity for the DNA and carefully scans the DNA base pairs leading to a low diffusion constant, while in state 2 the affinity is much reduced leading to a faster diffusion, however, in this state the TF cannot find its target. We showed here that using a switching process, the search for a target is faster, although the TF diffuses most of the time in state 2. This non-intuitive result further reveals that the effective binding rate cannot be assumed to be proportional to the fractional time spent in state 1, contrary to [56]. The second application illustrates our finding that a diffusing ligand can quickly activate a target, although it spends almost all of its time in a state where it has no affinity for the target. This is relevant if a ligand needs to activate a target in a state where it is also prone to degradation. We found that a ligand can largely avoid degradation and still perform a fast target activation by switching between two states such that it spends most of its time in the state 2 , where it cannot be degraded nor activate the target.

\section{Acknowledgments}

This research was supported by an ERC-starting grant and a HFSP research grant.

\section{Appendix. One-dimensional calculation}

When $\Omega=(0, L)$ is the one-dimensional interval with an absorbing boundary at $x=0$ in state 1 , and reflecting boundary 
at $x=L$, using the scaled variables,

$$
\begin{gathered}
\hat{x}=\frac{x}{L}, \quad l_{1}=\frac{k_{12} L^{2}}{D_{1}}, \quad l_{2}=\frac{k_{21} L^{2}}{D_{2}}, \\
\kappa=\frac{D_{1}}{D_{2}}, \quad v_{1}(\hat{x})=\frac{D_{1}}{L^{2}} u_{1}(x, 1), \\
v_{2}(\hat{x})=\frac{D_{1}}{L^{2}} u_{1}(x, 2) .
\end{gathered}
$$

Equation (10) is given by

$$
\begin{gathered}
v_{1}^{\prime \prime}(\hat{x})-l_{1}\left(v_{1}(\hat{x})-v_{2}(\hat{x})\right)=-1 \\
v_{2}^{\prime \prime}(\hat{x})+l_{2}\left(v_{1}(\hat{x})-v_{2}(\hat{x})\right)=0,
\end{gathered}
$$

with boundary conditions $v_{1}(0)=v_{1}^{\prime}(1)=v_{2}^{\prime}(0)=v_{2}^{\prime}(1)=$ 0 . Integrating the second equation over the scaled interval $(0,1)$ shows that the spatial averages satisfy the relationship (see equation (12))

$$
v_{1}=\int_{0}^{1} v_{1}(\hat{x}) \mathrm{d} \hat{x}=\int_{0}^{1} v_{2}(\hat{x}) \mathrm{d} \hat{x}=v_{2},
$$

and integration of the first equation provides the compatibility condition $v_{1}^{\prime}(0)=1$. When $l_{1}=0$, the solution $v_{1}(\hat{x})$ is the scaled mean first passage time

$$
\tau(\hat{x})=\frac{1}{2} \hat{x}(2-\hat{x})
$$

with spatial average

$$
\hat{\tau}=\int_{0}^{1} \tau(\hat{x}) \mathrm{d} \hat{x}=\frac{1}{3} .
$$

To proceed in deriving the solution of equations (A.2) we sum the two equations and obtain $v_{1}^{\prime \prime}(\hat{x})+\frac{l_{1}}{l_{2}} v_{2}^{\prime \prime}(\hat{x})=-1$, for which the solution is

$$
v_{1}(\hat{x})+\frac{l_{1}}{l_{2}} v_{2}(\hat{x})=\hat{\tau}(\hat{x})-\hat{\tau}+\left(1+\frac{l_{1}}{l_{2}}\right) v_{1},
$$

where we used the boundary conditions for $v_{1}(\hat{x})$ and $v_{2}(\hat{x})$ and $v_{1}=v_{2}$. By inserting $v_{2}(\hat{x})$ as a function of $v_{1}(\hat{x})$ into the first equation in (A.2) we find that $\psi(\hat{x})=v_{1}(\hat{x})-v_{1}$ satisfies

$$
\psi^{\prime \prime}(\hat{x})-\left(l_{1}+l_{2}\right) \psi(\hat{x})=-1-l_{2}(\hat{\tau}(\hat{x})-\hat{\tau}),
$$

with the boundary conditions $\psi^{\prime}(0)=1$ and $\psi^{\prime}(1)=0$. Using the variation of constant method we obtain the solution

$$
\begin{aligned}
\psi(\hat{x}) & =\frac{l_{2}}{l_{1}+l_{2}}(\hat{\tau}(\hat{x})-\hat{\tau})+\frac{l_{1}}{l_{1}+l_{2}} \\
\times & \left(\frac{\cosh \sqrt{l_{1}+l_{2}}-\cosh \left(\sqrt{l_{1}+l_{2}}(1-\hat{x})\right)}{\sqrt{l_{1}+l_{2}} \sinh \sqrt{l_{1}+l_{2}}}\right. \\
& \left.-f\left(l_{1}+l_{2}\right)\right)
\end{aligned}
$$

where the function

$$
f(x)=\frac{\operatorname{coth} \sqrt{x}}{\sqrt{x}}-\frac{1}{x}
$$

is monotonically decreasing from $\hat{\tau}=1 / 3$ at $x=0$ towards 0 for $x \rightarrow \infty$. Thus, since $v_{1}=-\psi(0)$ and $v_{1}(\hat{x})=$ $\psi(\hat{x})-\psi(0)$, we obtain

$$
\begin{gathered}
u_{1}(x, 1)=\frac{L^{2}}{D_{1}} v_{1}(\hat{x})=\frac{L^{2}}{D_{1}}\left(\frac{l_{2}}{l_{1}+l_{2}} \hat{\tau}(\hat{x})+\frac{l_{1}}{l_{1}+l_{2}}\right. \\
\left.\times \frac{\cosh \sqrt{l_{1}+l_{2}}-\cosh \left(\sqrt{l_{1}+l_{2}}(1-\hat{x})\right)}{\sqrt{l_{1}+l_{2}} \sinh \sqrt{l_{1}+l_{2}}}\right) \\
=\frac{l_{2}}{l_{1}+l_{2}} \tau_{1}(x)+\frac{L^{2}}{D_{1}} \frac{l_{1}}{l_{1}+l_{2}} \\
\times \frac{\cosh \sqrt{l_{1}+l_{2}}-\cosh \left(\sqrt{l_{1}+l_{2}} \frac{L-x}{L}\right)}{\sqrt{l_{1}+l_{2}} \sinh \sqrt{l_{1}+l_{2}}} \\
u_{1}(1)=\frac{L^{2}}{D_{1}} v_{1}=\tau_{1}-\frac{l_{1}}{l_{1}+l_{2}}\left(\tau_{1}-\frac{L^{2}}{D_{1}} f\left(l_{1}+l_{2}\right)\right)
\end{gathered}
$$

where $\tau_{1}(x)=\frac{L^{2}}{D_{1}} \hat{\tau}(\hat{x})$ and $\tau_{1}=\frac{L^{2}}{D_{1}} \hat{\tau}$.

\section{References}

[1] Berg H C and Purcell M 1977 Biophys. J. 20193

[2] Shoup D and Szabo A 1982 Biophys. J. 4033

[3] Zwanzig R 1990 Proc. Natl Acad. Sci. USA 875856

[4] Zwanzig R and Szabo A 1991 Biophys. J. 60671

[5] Hildebrand J 1995 Proc. Natl Acad. Sci. USA 9267

[6] Schuss Z, Singer A and Holcman D 2007 Proc. Natl Acad. Sci. USA 10416098

[7] Szabo A, Schulten K and Schulten Z 1980 J. Chem. Phys. 724350

[8] Schulten K, Schulten Z and Szabo A 1981 J. Chem. Phys. 744426

[9] Perico A and Battezzati M 1981 J. Chem. Phys. 754430

[10] Wilemski G and Fixman M 1973 J. Chem. Phys. 584009

[11] Collins F C and Kimball G E 1949 J. Colloid Sci. 4425

[12] Holcman D and Schuss Z 2005 J. Chem. Phys. 122114710

[13] Ward M and Keller J 1993 SIAM J. Appl. Math. 53770

[14] Ward M, Henshaw W and Keller J 1993 SIAM J. Appl. Math. 53799

[15] Kolokolnikov T, Titcombe M and Ward M 2005 Eur. J. Appl. Math. 16161

[16] Grigoriev I V, Makhnovskii Y A, Berezhkovskii A M and Zitserman V Y 2002 J. Chem. Phys. 1169574

[17] Straube R, Ward M and Falcke M 2007 J. Stat. Phys. 129377

[18] Singer A, Schuss Z, Holcman D and Eisenberg B 2006 J. Stat. Phys. 122437

[19] Singer A, Schuss Z and Holcman D 2006 J. Stat. Phys. 122491

[20] Singer A, Schuss Z and Holcman D 2008 Phys. Rev. E 78051111

[21] Reingruber J, Abad E and Holcman D 2009 J. Chem. Phys. 130094909

[22] Lagache T, Dauty E and Holcman D 2009 Phys. Rev. E 79011921

[23] Lagache T, Dauty E and Holcman D 2009 Curr. Opin. Microbiol. 12439

[24] Holcman D and Triller A 2006 Biophys. J. 912405

[25] Holcman D and Schuss Z 2004 J. Stat. Phys. 117975

[26] Reingruber J and Holcman D 2009 Phys. Rev. E 79030904

[27] Ptashne M 2004 Genetic Switch: Phage Lambda Revisited (New York: Cold Spring Harbor)

[28] Barrandon C, Spiluttini B and Bensaude O 2008 Biol. Cell 10083

[29] Barrandon F, Bonnet C, Nguyen V, Labas V and Bensaude O 2007 Mol. Cell. Biol. 276996

[30] Berg T 2008 Curr. Opin. Chem. Biol. 12464 
[31] Majmudar C and Mapp A 2005 Curr. Opin. Chem. Biol. 9467

[32] Szabo A, Shoup D, Northrup S and McCammon J $1982 \mathrm{~J}$. Chem. Phys. 774484

[33] Zwanzig R 1992 J. Chem. Phys. 973587

[34] Zhou H and Szabo A 1996 J. Phys. Chem. 1002597

[35] Berezhkovskii A, Yang D, Sheu S and Lin S 1996 Phys. Rev. E 544462

[36] Doering C 2000 Stochastic Processes in Physics, Chemistry, and Biology (Lecture Notes in Physics vol 557) (Berlin: Springer) pp 316-26

[37] Makhnovskii Y, Berezhkovskii A, Sheu S, Yang D, Kuo J and Lin S 1998 J. Chem. Phys. 108971

[38] Doering C and Gadoua J 1992 Phys. Rev. Lett. 692318

[39] Bier M and Astumian R 1993 Phys. Rev. Lett. 711649

[40] Bénichou O, Coppey M, Moreau M, Suet P and Voituriez R 2005 Phys. Rev. Lett. 94198101

[41] Bénichou O, Coppey M, Moreau M, Suet P and Voituriez R 2005 J. Phys.: Condens. Matter 17 S4275

[42] Bénichou O, Loverdo C, Moreau M and Voituriez R 2006 Phys. Rev. E 74020102

[43] Loverdo C, Bénichou O, Moreau M and Voituriez R 2008 Nat. Phys. 4134
[44] Loverdo C, Bénichou O, Moreau M and Voituriez R 2009 Phys. Rev. E 80031146

[45] Risken H 1996 The Fokker-Planck Equation (New York, LLC: Springer)

[46] Schuss Z 1980 Theory and Applications of Stochastic Differential Equations (Wiley Series in Probability and Statistics) (New York: Wiley)

[47] Bénichou O, Coppey M, Moreau M and Voituriez R 2006 Europhys. Lett. 75349

[48] Gillespie D T 1976 J. Comput. Phys. 22403

[49] Elf J, Li G and Xie X 2007 Science 3161191

[50] Wang Y, Austin R and Cox E 2007 Science 97048302

[51] Von Hippel P and Berg O G 1989 J. Biol. Chem. 264675

[52] Winter R B, Berg O G and von Hippel P H 1989 Biochemistry 20 6961-77

[53] Slutsky M and Mirny L 2004 Biophys. J. 874021

[54] Malherbe G and Holcman D 2010 Phys. Lett. A 374466

[55] Hu L, Grosberg A and Shklovskii B 2006 Biophys. J. 902731

[56] Hu L, Grosberg A and Bruinsma R 2008 Biophys. J. 951151

[57] Bénichou O, Kafri Y, Sheinman M and Voituriez R 2009 Phys. Rev. Lett. 103138102 